\title{
INTEGRATED PLASMA CONTROL FOR ADVANCED TOKAMAKS
}

\author{
by \\ D.A. HUMPHREYS, J.R. FERRON, R.D. JOHNSON, \\ J.A. LEUER, B.G. PENAFLOR, M.L. WALKER, \\ A.S. WELANDER, R.R. KHAYRUTDINOV, \\ V. DOKOUKA, D.H. EDGELL, and C.-M. FRANSSON
}




\section{DISCLAIMER}

This report was prepared as an account of work sponsored by an agency of the United States Government. Neither the United States Government nor any agency thereof, nor any of their employees, makes any warranty, express or implied, or assumes any legal liability or responsibility for the accuracy, completeness, or usefulness of any information, apparatus, product, or process disclosed, or represents that its use would not infringe privately owned rights. Reference herein to any specific commercial product, process, or service by trade name, trademark, manufacturer, or otherwise, does not necessarily constitute or imply its endorsement, recommendation, or favoring by the United States Government or any agency thereof. The views and opinions of authors expressed herein do not necessarily state or reflect those of the United States Government or any agency thereof. 
GA-A24494

\title{
INTEGRATED PLASMA CONTROL FOR ADVANCED TOKAMAKS
}

\author{
by \\ D.A. HUMPHREYS, J.R. FERRON, R.D. JOHNSON, \\ J.A. LEUER, B.G. PENAFLOR, M.L. WALKER, \\ A.S. WELANDER, R.R. KHAYRUTDINOV, ${ }^{*}$ \\ V. DOKOUKA, ${ }^{*}$ D.H. EDGELL, $\dagger$ and C.-M. FRANSSON $\ddagger$ \\ ${ }^{*}$ TRINTI Laboratory \\ tFarTech, Inc. \\ ‡Chalmers University
}

This is a preprint of a paper presented at the 20th IEEE/NPSS Symposium on Fusion Engineering, San Diego, California, October 14-17, 2003 and to be published in Fusion Science and Technology.

\author{
Work supported by \\ the U.S. Department of Energy \\ under Contract No. DE-AC03-99ER54463
}

GENERAL ATOMICS PROJECT 30033 MARCH 2004 


\title{
Integrated Plasma Control for Advanced Tokamaks
}

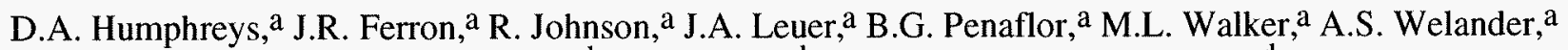 \\ R.R. Khayrutdinov, ${ }^{b}$ V. Dokouka, ${ }^{b}$ D.H. Edgell, ${ }^{c}$ C.-M. Franssond \\ $a_{\text {General Atomics, P.O. Box 85608, San Diego, California } 92186-5608}$ \\ ${ }^{b}$ Triniti Laboratory, Troitsk, Russia \\ cFar-Tech, Inc., San Diego, CA \\ $d_{\text {Chalmers Univ., Gothenburg, Sweden }}$
}

\begin{abstract}
Advanced tokamaks (AT) [1] are distinguished from conventional tokamaks by their high degree of shaping, achievement of profiles optimized for high confinement and stability characteristics, and active stabilization of MHD instabilities to attain high values of normalized beta and confinement. These high performance fusion devices thus require accurate regulation of the plasma boundary, internal profiles, pumping, fueling, and heating, as well as simultaneous and well-coordinated MHD control action to stabilize such instabilities as tearing modes and resistive wall modes. Satisfying the simultaneous demands on control accuracy, reliability, and performance for all of these subsystems requires a high degree of integration in both design and operation of the plasma control system in an advanced tokamak. The present work describes the approach, benefits, and progress made in integrated plasma control with application examples drawn from the DIII-D tokamak. The approach includes construction of plasma and system response models, validation of models against operating experiments, design of integrated controllers which operate in concert with one another as well as with supervisory modules, simulation of control action against off-line and actual machine control platforms, and iteration of the design-test loop to optimize performance.
\end{abstract}

\section{INTRODUCTION}

While all magnetic fusion confinement systems have close couplings among configuration, operating point, transport, and stability, the degree and importance of these couplings is critical in achieving the advanced tokamak regime. Fig. 1 illustrates many of the important physics interactions within AT plasmas. Central to sustaining AT performance is maintaining plasma equilibria in a highly optimized shape, and current profiles in a specific state characterized by good bootstrap current alignment and actively driven noninductive current. At the same time, internal transport barriers must be maintained, and a variety of MHD instabilities must be suppressed. Key instabilities which can limit the achievable normalized beta include the neoclassical tearing mode (NTM) [2] and the resistive wall mode (RWM) [3]. Many of the internal physics elements shown in Fig. 1 are strongly affected by such instabilities. Because the use of any individual actuator in the system can strongly affect more than one physics aspect, the nature of these responses must be built into models of the system in order to design high performance controllers. In order to provide the necessary controller reliability, these highly coupled models must also be

ADVANCED TOKAMAK NONLINEAR TRANSPORT COUPLINGS

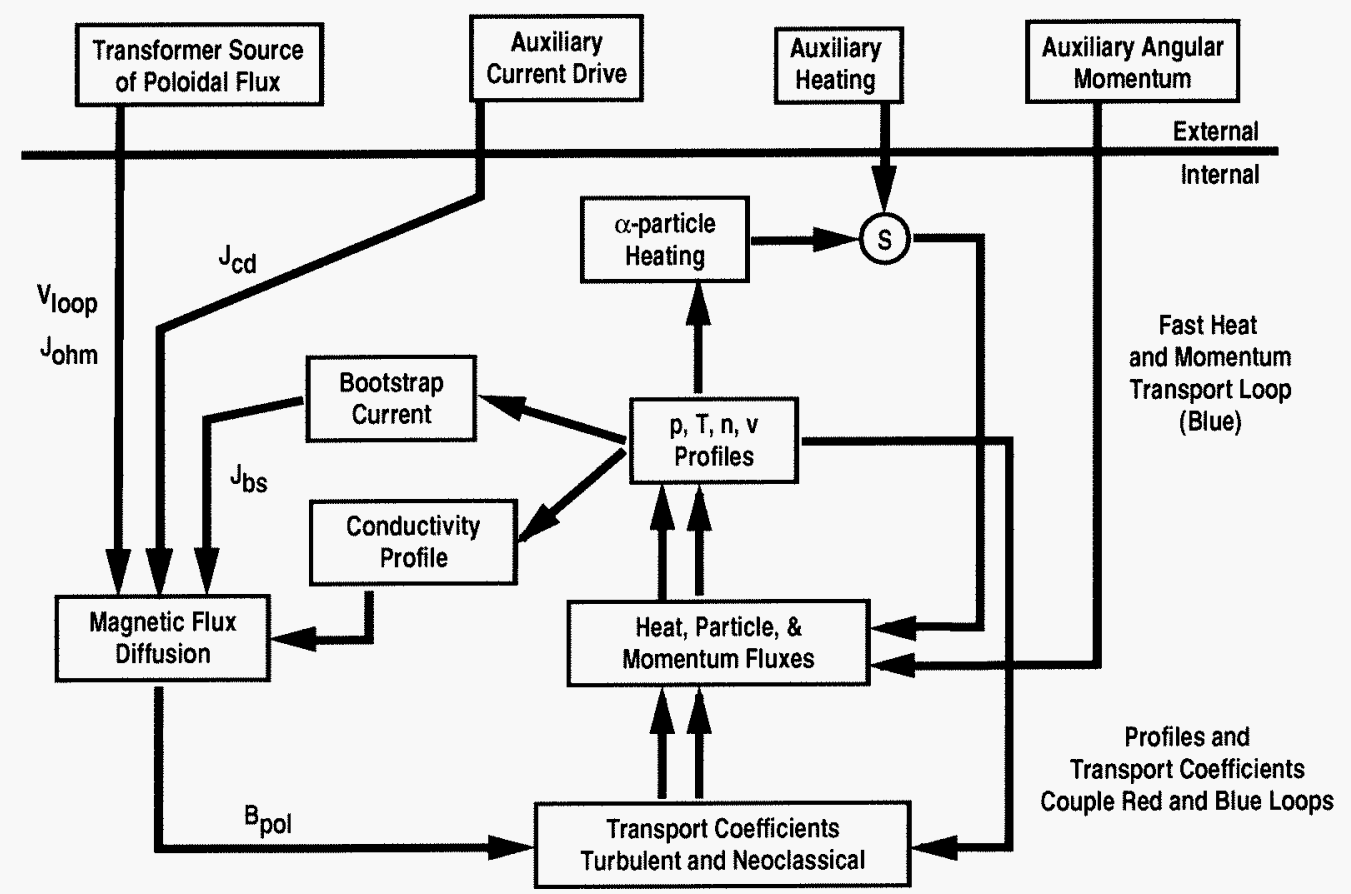

Slow Magnetic Flux Transport Loop (Red)

Fig. 1. Some of the highly-coupled physics effects in advanced tokamaks. 
validated extensively across a broad range of operating regimes.

Highly accurate dynamic models of system responses which have been validated against experimental data allow high reliability control design to be done. Linear multivariable design techniques, for example, allow explicit design for desired performance, and enable the determination of controllers without the necessity of empirical optimization which requires machine operation time. Such approaches as linear optimal design [4] allow weighting of relative importance of actuator power or control errors to achieve the desired balance of control emphasis, while $\mathrm{H}_{\infty}[5]$ and other robust design methods allow shaping of control loop response while ensuring specified robustness to such nonideal effects as model uncertainties. For present-day devices, the use of such design techniques can greatly reduce the need for machine time traditionally dedicated to control optimization, and can even allow determination of high-reliability controllers prior to ever producing the target equilibrium experimentally.

Since control design is typically performed using linear and often further-simplified system models, controller performance must be tested against more realistic simulations prior to implementation. Such simulations are particularly important for testing control performance in the presence of actuator saturation, power supply nonlinearities, and other complex system details. Simulations are also important for testing performance in the presence of realistic nonlinear plasma responses, particularly when the plasma response diverges significantly from the linearized design model response. Two kinds of simulation are essential for integrated plasma control: "offline" testing of controllers implemented in a closed-loop simulation, and "hardware-in-the-loop" testing of the PCS controller implementation running on actual realtime computers communicating with the simulation. This latter capability allows verification of both the correctness of the controller implementation in the PCS and the performance of the algorithm when running on actual real-time computers against a realistic system response.

\section{CONTROL ISSUES IN ADVANCED TOKAMAKS}

Advanced tokamaks are highly coupled systems which operate in very narrow windows of physics operating space in order to achieve the high performance promised by the configuration. In a high performance advanced tokamak, accurate regulation of the plasma boundary, internal profiles, pumping, fueling, and heating must be well coordinated with MHD control action to stabilize such instabilities as tearing modes and resistive wall modes. Sophisticated monitors of the operational regime must provide detection of off-normal conditions and trigger appropriate safety responses with acceptable levels of reliability.

Operating point control, including high-demand shaping and axisymmetric stability control, profile control, heating and density control must be highly optimized in order to make best use of limited auxiliary heating and current drive systems. AT reactors must maintain this narrow operating window in the presence of alpha particle heating and the potential for thermal runaway. Constraints on steady state shape regulation are more severe for a reactor, which can tolerate only momentary excursions from the nominal equilibrium, and are often in partial conflict with the more demanding AT configuration. For example, high performance AT equilibria must have high elongation, leading to high vertical instability growth rates. The natural response to fluctuations in such a plasma typically operating near the ideal vertical stability limit produces high frequency fluctuating fields at the poloidal field coils. However, the superconducting PF coils of a reactor place strong restrictions on the amplitude of these fluctuating fields because of the AC loss heating which can quench the coils.

Strongly coupled to the operating point control is the need to provide passive and/or active MHD stability control in an AT. Closed loop control of selected profile characteristics can maintain equilibria at operating points which are stable to various MHD modes. For example, local current drive or heating may allow maintenance of $\Delta^{\prime}$ sufficiently negative to stabilize the NTM [2]. Even in the presence of unstable equilibria, many modes can be actively suppressed as well. For example, driving current at the location of NTM islands can completely stabilize these modes. Alignment of the current drive deposition region with the location of the island can require plasma positioning, launcher mirror control, or toroidal field control with precision and accuracy corresponding to better than $0.5 \%$ of the minor radius in an AT reactor. Satisfying such dynamic control constraints in the presence of typical disturbances and noise in an AT plasma requires taking into account the effect of various control loops on the NTM control. In addition, the position control must be integrated with the NTM control in order to maintain alignment after suppression of the mode. Other MHD instabilities must also be stabilized in order to access the highest AT performance regimes. Stabilization of the RWM in particular can produce large gains in achievable normalized beta [3]. While the coupling between the nonaxisymmetric RWM system and axisymmetric control can be made less significant by connecting diagnostics and control coils in explicitly $n=$ odd configurations, other nonaxisymmetric phenomena such as ELMs remain closely coupled to RWM control systems and must be accounted for in modeling and design. Furthermore, variations in the equilibrium, e.g., higher $\beta$ and sustained rotation which result from successful stabilization of the RWM can lead to different dynamics which should then be accounted for in the RWM control algorithm in order to provide sustained high robustness stabilization.

Another highly-coupled category of control includes offnormal event response, including detection of impending disruptions, determination and execution of corrective action (where possible), or execution of a mitigating response (where correction/recovery is not possible). The system responsible for off-normal detection and response must be thoroughly integrated with many other control subsystems in order to provide effective and appropriate action coordinated with other plasma control responses. Such responses must also be well-integrated with the overall safety and supervisory system.

\section{INTEGRATED PLASMA CONTROL}

"Integrated plasma control" has come to refer to two related but different concepts. The first refers to a form of control scheme in which many different quantities are controlled simultaneously by many actuators using control algorithms which take into account the coupled interactions across many areas of physics (for example, axisymmetric discharge characteristics and nonaxisymmetric MHD instabilities). The second concept refers to a methodology for systematically developing and confirming the performance of 
such multivariable controllers which must function with high reliability in a highly coupled environment. This approach is summarized schematically in Fig. 2. Physics understanding produces models of system response, which are used in the design of control algorithms. Typically models used in control design must be linearized or otherwise simplified from the full nonlinear physical response of the system. Linearized models allow use of the rich technology of linear multivariable control design to produce robust and/or cost-functional optimized controllers. Control designs are then tested against detailed system simulations, which include important nonlinearities such as actuator or sensor saturation, and allow iterative improvement of controller performance. The optimized controller is implemented in the control software running on the actual control computer hardware, which is then itself tested against the detailed system simulation. This final test confirms that the implementation is correct, and that no unforeseen functional details of the control hardware will adversely affect control operation. Experimental results are used at the beginning of the process and continuously through operation to validate the models used for design and simulation.

In the discussions following the emphasis will be on design approach, although much will also apply to the closelyrelated issues of integrated plasma control schemes themselves.

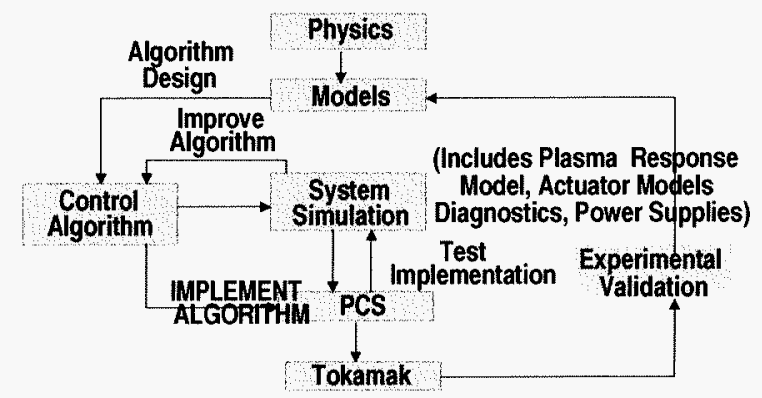

Fig. 2. Elements of the integrated plasma control approach.

\section{A. Axisymmetric System Models and Validation}

Plasma control which integrates the various elements required by an AT must make use of many different system models, describing among other physical characteristics, the axisymmetric equilibrium and profile evolution of the plasma in response to conductor currents, current drive, heating, and particle source actuators. The field of basic equilibrium evolution modeling with constrained profiles has been very successful over the past decade. Beginning with simple current- or flux-conserving rigid plasma displacements and continuing to nonrigid and resistive plasmas, such models have successfully reproduced detailed plasma evolution in several experiments as well as enabling design of multivariable controllers which have been tested successfully in experimental devices [6,7].

Axisymmetric plasma response models are typically based on a combination of Faraday's law applied to toroidal conductor circuits and perturbed plasma equilibria or force balance equations [8]. The general form of the resulting conductor circuit equation for linearized plasma displacements is

$M_{s s} \dot{I}_{s}+R_{s s} I_{s}+M_{s p} \dot{I}_{p}+\frac{\partial \psi_{s}}{\partial \xi_{R}} \dot{\xi}_{R}+\frac{\partial \psi_{s}}{\partial \xi_{R}} \dot{\xi}_{R}=V_{s}$, and a resistive plasma can be included in the overall circuit equation via

$$
M_{p p} \dot{I}_{p}+R_{p p} I_{p}+M_{s p} \dot{I}_{s}+\frac{\partial \psi_{s}}{\partial \xi_{R}} \dot{\xi}_{R}+\frac{\partial \psi_{s}}{\partial \xi_{R}} \dot{\xi}_{R}=V_{s}
$$

while the linearized radial and vertical force balance at each fluid element (neglecting plasma inertia) are given respectively by

$$
\begin{aligned}
& \delta F_{R}=0=\frac{\partial F_{R}}{\partial I_{s}} I_{s}+\frac{\partial F_{R}}{\partial I_{p}} I_{p}+\frac{\partial F_{R}}{\partial \xi_{R}} \xi_{R}+\frac{\partial F_{R}}{\partial \xi_{z}} \xi_{z}+\frac{\partial F_{R}}{\partial \beta_{p}} \beta_{p} \\
& \delta F_{Z}=0=\frac{\partial F_{Z}}{\partial I_{s}} I_{s}+\frac{\partial F_{Z}}{\partial I_{p}} I_{p}+\frac{\partial F_{Z}}{\partial \xi_{R}} \xi_{R}+\frac{\partial F_{Z}}{\partial \xi_{Z}} \xi_{z}+\frac{\partial F_{Z}}{\partial \beta_{p}} \beta_{p}
\end{aligned}
$$

where $I_{S}$ is the vector of perturbed conductor currents, $I_{p}$ is the vector of perturbed plasma fluid element currents, $\psi_{s}$ is the vector of perturbed flux at conductors, $\xi_{R}$ and $\xi_{Z}$ are plasma fluid element $\mathrm{R}$ and $\mathrm{Z}$ displacement vectors respectively, and $\beta_{\mathrm{p}}$ is a perturbed poloidal beta (scalar or vector representing local values). Combining Eqs. (1) through (4) produces a cumulative circuit equation describing toroidal conductors and plasma fluid element currents, which includes the effect of nonrigid plasma displacement under the massless or quasiequilibrium assumption. The plasma current vector $\mathrm{I}_{\mathrm{p}}$ is often determined by either fixing it (constant current approximation), varying the $I_{p}$ values in order to exactly conserve flux at each element (exact ideal MHD approximation), or conserving some measure of average flux over the plasma (approximate resistive plasma approximation). Explicitly including a plasma circuit equation such as Eq. (2) which allows each fluid element to vary according to the local neoclassical resistivity accounts for resistive magnetic flux diffusion through the plasma even more accurately, provided the plasma resistivity is sufficiently well known. Diffusion of currents driven in the plasma edge region occurs on a timescale much shorter than the core plasma current. Therefore, using core plasma current diffusion alone may ignore central dynamics of the plasma edge. In DIII-D, driven edge plasma region currents at $\mathrm{T}_{\mathrm{e}}$ $100-500 \mathrm{eV}$ typically diffuse with time constants on the order of $5-20 \mathrm{~ms}$, comparable to shape and position control timescales. Ignoring edge current dynamics in the plasma model can therefore have a dramatic effect on shape and stability control performance.

\section{B. Offline Simulations}

Simulations are an essential part of the integrated plasma control development process. Detailed simulations constructed of well-validated modules describing the key elements of the full system allow testing of control algorithms prior to implementation in the actual tokamak PCS. This "offline" simulation is particularly important when the control design is carried out with linear or otherwise simplified models, yet the final system contains important nonlinearities and other complexities not well-described by the design-level models. Fig. 3 shows a schematic of a Simulink implementation of a highly integrated simulation, including axisymmetric control elements (plasma-conductor models in particular), MHD control elements (NTM and RWM), and disruption 


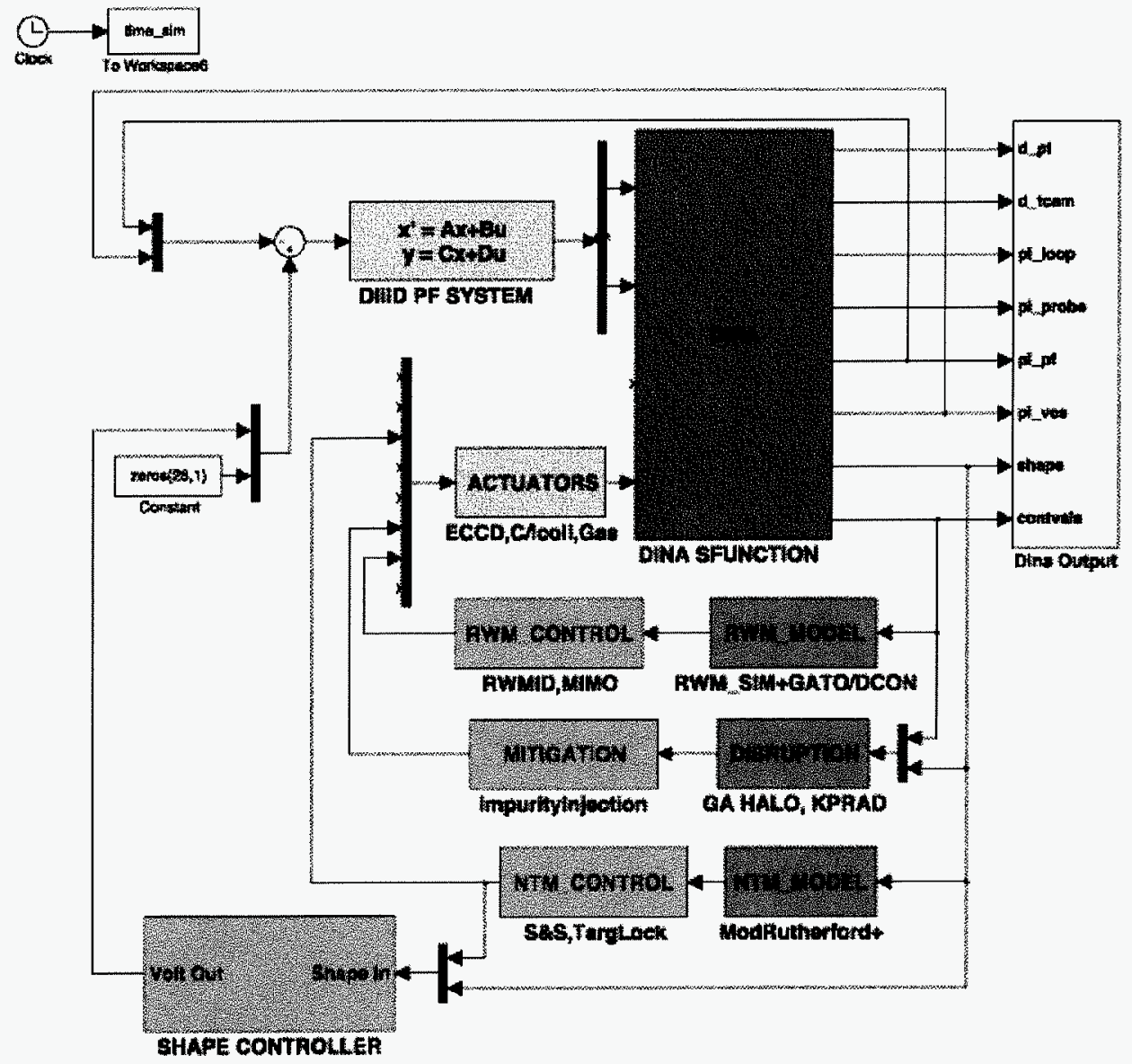

Fig. 3. Simulink implementation of integrated system model for simultaneous simulation of multiple control modules.

detection/mitigation elements. Each loop contains detailed representations of both controllers and physics response models, and can include sophisticated logic as well as mathematical signal processing operations.

\section{Hardware-in-the-Loop Simulations}

Another indispensable element of integrated plasma control development is the "hardware-in-the-loop" testing process. This process involves direct testing of the actual PCS hardware and software implementation of control algorithms against detailed simulations similar to those used for offline simulation. This process is summarized schematically in Fig. 4. The PCS system routinely operates in direct communication with tokamak diagnostics while sending actuator control signals to the various control subsystems. The simulation is configured to provide modeled diagnostic signals exactly paralleling the set of signals obtained from the tokamak in experimental operations. The PCS then performs its realtime calculations and outputs the corresponding actuator commands. The event timing is managed between simulation and PCS to precisely match the timing experienced in experimental operations as well. This allows testing of such hardware-dependent characteristics as calculation delay, data transfer latency, signal-port alignment, filter performance, and network communication. The hardware-in-the-loop results are compared with off-line closed-loop simulations of the sasme control algorithm. Using this final testing step provides high confidence in implementation and controller performance

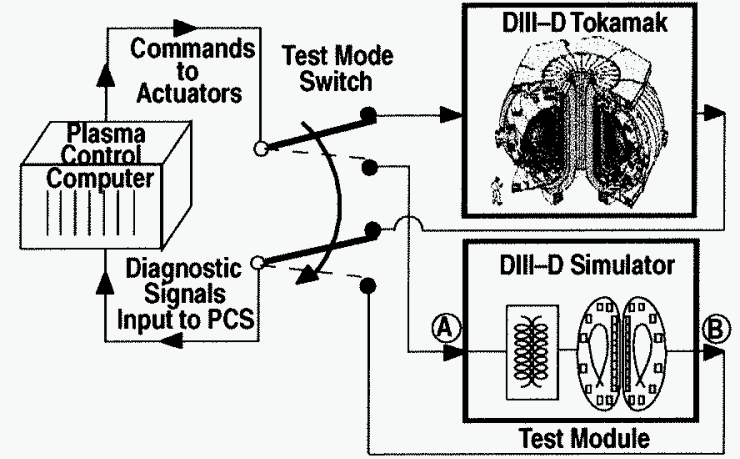

Fig. 4. Hardware in the Loop schematic. Actual plasma control computer hardware and controller implementations can be operated against the DIII-D simulator test module instead of the tokamak itself.

under actual machine operational conditions well before experimental execution.

\section{INTEGRATED PLASMA CONTROL IN THE DIII-D ADVANCED TOKAMAK PROGRAM}

A principal focus of the DIII-D experimental program is achievement and performance extension of the advanced tokamak regime, requiring highly integrated and flexible plasma control. The DIII-D PCS [9] provides a powerful environment for implementation and programming of control 
algorithms, as well as an architecture which can support realtime use of many cpus communicating on a fast network. The interface and code development environment allows definition of any desired control categories and discharge phases, realtime switching between one control phase and another, and user-friendly programming of waveforms to specify the desired discharge control configuration. The multicpu architecture allows simultaneous execution of many computationally intensive control algorithms along with coordinated event-handling to manage highly-coupled actuator commands and rapid realtime responses to changing discharge conditions.

\section{A. Axisymmetric Modeling and Simulation for Integrated Control Design and Testing}

A complete suite of design, analysis and simulation tools has been developed to better implement axisymmetric controllers for DIII-D. The collection of software consists of models of DC power supplies, fast switched power supplies, field shaping and ohmic heating coils, passive vacuum vessel, linear plasma response, data filters, magnetic diagnostics and $\mathrm{A} / \mathrm{D}$ and $\mathrm{D} / \mathrm{A}$ signal converters. These validated models provide a basis for controller design and allow detailed simulation of the DIII-D system for use in both offline and hardware-in-the-loop testing of controllers. A schematic of this simulation environment is shown in Fig. 5, illustrating the level of detail included. Either linearized plasma models such as that described in Section III.A or a full nonlinear 1-1/2D resistive MHD model such as the DINA [10] code can be used for the plasma block. These models have been validated against DIII-D experimental data in significant detail [11].

\section{B. Integrated Modeling and Simulation for NTM Control Development}

NTM stabilization provides an excellent example of closely-coupled stability and equilibrium control in which the integrated plasma control approach was successfully applied to performance enhancement in DIII-D. The DIII-D gyrotron system [12] presently allows up to $3 \mathrm{MW}$ of electron cyclotron current drive (ECCD) to be driven at the second harmonic resonance. Alignment of this deposition region with the location of an NTM island allows replacement of the bootstrap current deficit which characterizes the instability. Replacing the bootstrap current stabilizes the mode, allowing achievement of higher values of normalized beta than achievable in the presence of saturated islands.

The NTM control system in DIII-D makes use of several coupled algorithms, among which are the Search and Suppress and Active Tracking routines. Each of these can affect one of three different island/ECCD alignment control quantities: the plasma major radial position, the toroidal field, or the plasma vertical position. The operation mode which has been most successfully and routinely applied to date is a combined Search/Suppress and Active Tracking mode. When the control phase is enabled in this mode, the control algorithm fixes the selected control quantity for a specified "dwell time" to determine whether the degree of alignment is sufficient to suppress the mode. If the mode is not sufficiently suppressed during the dwell, the algorithm executes a "search" by incrementing the control quantity by a specified amount, freezing the control quantity for another dwell time, and examining the resulting effect on the mode. This search/dwell/search sequence continues until a specified limit is reached, and the search reverses the sign of the control quantity increment. This process continues until sufficient alignment is detected and the mode is suppressed below the specified amplitude threshold.

Once a sufficient alignment is detected and the mode is suppressed, the control quantity is nominally frozen, and the Active Tracking algorithm is engaged. This algorithm adjusts the control quantity to maintain alignment in the absence of the mode. The required adjustment is determined by a linear or nonlinear predictor calculation based on magnetic measurements.

Fig. 6 shows the first step in integrated plasma control process for NTM suppression: a comparison of the mode

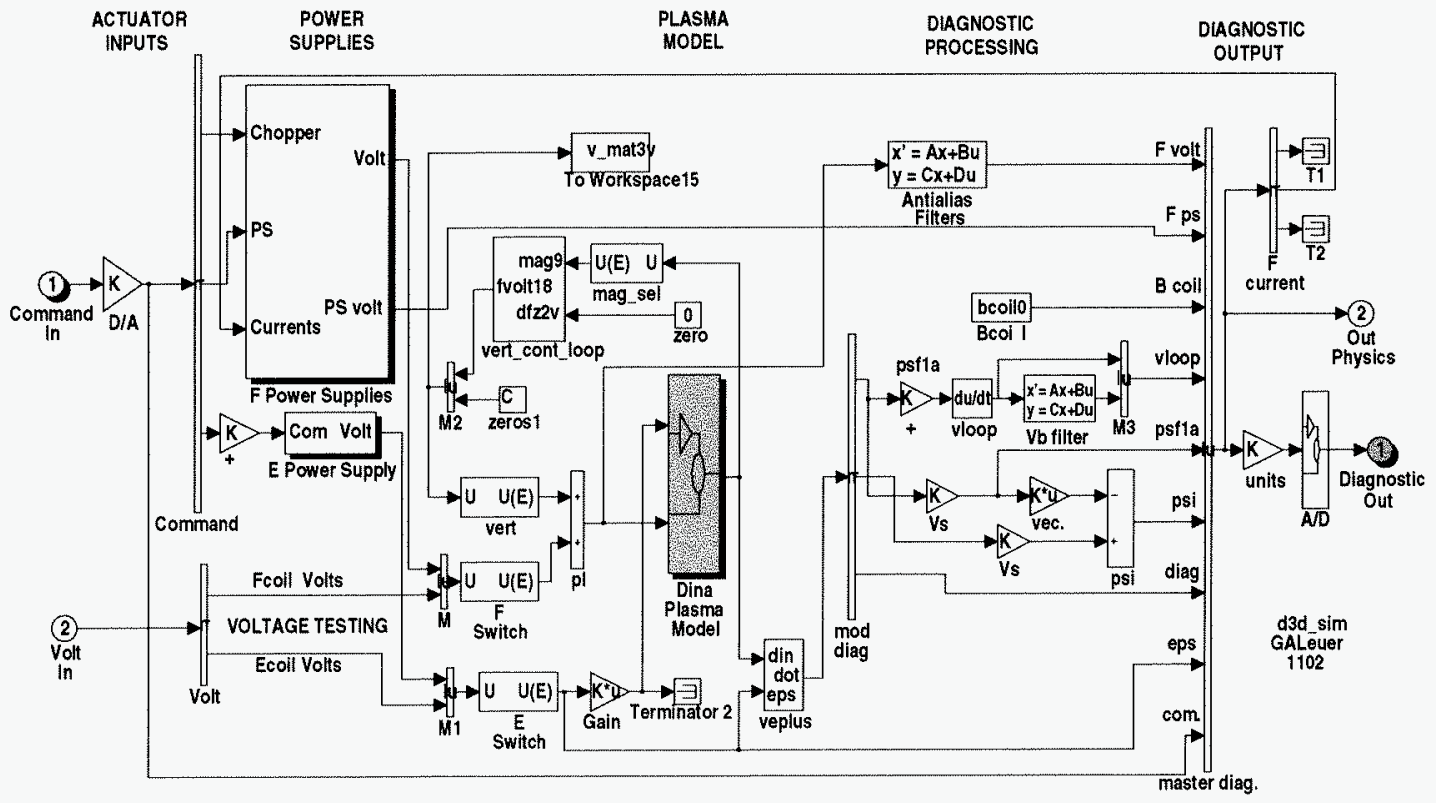

Fig. 5. Major elements of the DINA/DIII-D axisymmetric system simulator, used for both offline and hardware-in-the-loop simulation. 

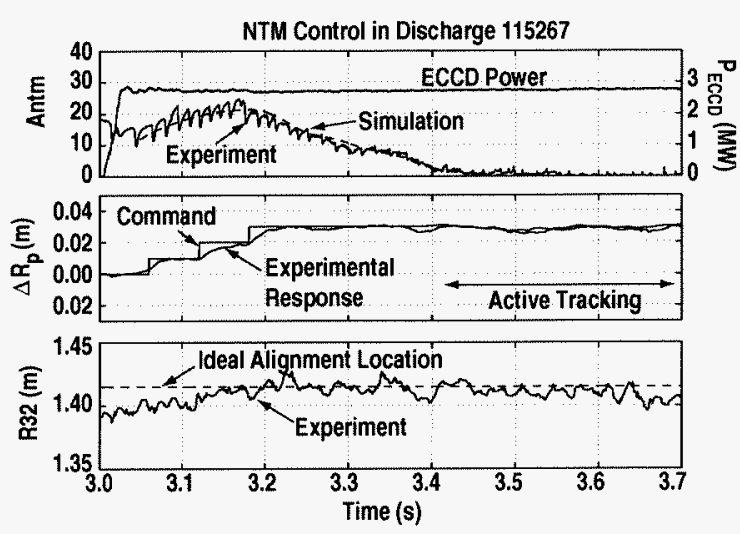

Fig. 6. NTM suppression experiment and simulation using design model of island response to ECCD application.

suppression model and the experimental response to variation in the degree of alignment between island and ECCD location (top frame). The model response, based on a simplified version of the modified Rutherford equation [2], shows sufficiently accurate representation of island response dynamics to allow for good control design. In the case shown, the plasma major radius (middle frame) was varied to adjust the alignment of the $q=1.5$ surface with the ECCD deposition location. Following suppression of the mode, the Active Tracking algorithm is enabled to compensate for variations in the $q=1.5$ surface due to changes in the current profile and poloidal beta. The signature of Active Tracking can be seen in the fluctuating major radius perturbations following suppression at $\mathrm{t} \sim 3.4 \mathrm{~s}$.

Fig. 6 also summarizes experimental results from use of the Search and Suppress with Active Tracking. The growth of the mode is slowed when the island and ECCD are misaligned by as much as $1.5-2 \mathrm{~cm}$. Adjustment of the major radius by the Search and Suppress produces sufficient alignment to fully suppress the mode within $200 \mathrm{~ms}$. After design and testing of the basic scheme, proper functioning of the algorithm requires specification of various parameters and thresholds to match the dynamic characteristics of the target equilibrium. Using integrated plasma control techniques, the system was designed with sufficient reliability to produce successful suppression and tracking of the evolution of the profile to maintain island/ECCD alignment the first time this integrated active suppression was attempted experimentally.

Use of Search and Suppress and Active Tracking algorithms have enabled full suppression of both $3 / 2$ and $2 / 1$ NTM's in DIII-D under full closed loop control without previous specification of the ideal alignment location. Suppression of the $3 / 2$ NTM has allowed operation at normalized beta values $-50 \%$ above the value achieved in the presence of the unsuppressed mode (from $\beta_{N}=2.3$ to $\beta_{N}=3.4$ ). This is achieved with Active Tracking modification of the major raidiu of several centimeters. The duration of increasedbeta operation is presently limited by the length of the gyrotron pulses.

\section{INTEGRATED PLASMA CONTROL AND ITTER}

The International Thermonuclear Experimental Reactor (ITER) device design poses unique challenges to control, owing primarily to its role as a burning plasma experiment. ITER also now has a significant AT mission, including operation beyond the no-wall beta limit with active RWM and NTM stabilization, high performance shaping, and active profile control. In addition to the demanding elements of longpulse AT operation, high performance AT scenarios in ITER will produce substantial alpha particle self-heating, further complicating the multivariable coupled control problem. Long pulse operations, extending many current diffusion times, also exacerbate the control problem by potentially introducing significant drift in the magnetic and other integrated diagnostic signals, which are key to accurate shape and profile control. In an ELMy $\mathrm{H}$-mode scenario, the ablation due to repetitive ELM heat pulses may significantly limit the lifetime of the divertor targets, requiring divertor cassette replacement at an unacceptably high rate. A high frequency of unmitigated disruptions will produce a similar ablation-limited divertor lifetime and require unacceptable replacement and maintenance schedules. Disruption detection, avoidance, correction, and mitigation are thus essential for operation of ITER.

The application of the integrated plasma control approach to ITER is still in its early stages. However, studies during the ITER EDA and beyond strongly suggest that detailed modeling and simulation will be important in finalizing control designs for commissioning prior to experimental operations $[13,14]$. Designs of supervisory systems for disruption response are also amenable to the approach.

\section{SUMMARY AND CONCLUSIONS}

The integrated plasma control approach provides a systematic method for developing high reliability control systems for advanced tokamaks. This approach is highly efficient, allowing development of high confidence, high performance control algorithms with greatly reduced need for experimental testing and optimization time. Elements of the approach are already being applied to tokamaks around the world, including DIII-D, as well as in design projects for next generation devices such as ITER. Present-day devices exploring the AT regime have produced sufficient physics understanding to enable accurate modeling of most of the critical response elements, highlighting the critical role of operating machines in the design of next-generation deviices. Many of the recent experimental successes in performance enhancement through sustained operation in advanced tokamak regimes have been made possible through detailed modeling, simulation, control design, and algorithm improvement performed prior to actual experimental use. Successful application of such coupled control algorithms in first-time use on operating devices has demonstrated the reliability and importance of the approach for next-generation device designs.

\section{ACKNOWLEDGEMENTS}

The authors are grateful for the kind contribution of Fig. 1 by Dr. P. Politzer of General Atomics. Work supported by U.S. Department of Energy under Contract No. DE-AC0399ER54463.

\section{REFERENCES}

[1] Ferron, J.R., et al, "Progress toward sustained highperformance advanced tokamak discharges in DIII-D," Proc. 29th Plasma Phys. and Contr. Fus. Conf., Montreux, Switzerland, June 2002, Vol. 26B, P1.060. 
[2] La Haye, R.J., et al., "Control of neoclassical tearing modes in DIII-D," Phys. of Plasmas 9 (2002) 2051.

[3] Garofalo, A.M., et al., "Stabilization of the external kink and control of the resistive wall mode in tokamaks," Phys. Plasmas 6 (1999) 1893.

[4] Maciejowski, J.M., "Multivariable feedback design," Addison-Wesley, Wokingham, England, 1989.

[5] McFarlane, D.C., Glover, K., "Robust controller design using normalized coprime factor plant descriptions," Springer-Verlag, Lect. Notes in Control and Inf. Sci. 138 (1989).

[6] Walker, M.L., Humphreys, D.A., Leuer, J.A., Ferron, J.A., Penaflor, B.G., "Initial implementation of modelbased multivariable control on DIII-D," Proc. $21 \mathrm{st}$ Symp. on Fus. Technol., Madrid, Spain, (2000).

[7] Ariola, M. Ambrosino, G., Lister, J.B., Pironti, A., Villone, F., Vyas, P., "A modern plasma controller tested on the TCV tokamak," Fus. Tech. 36 (1999) 126.

[8] Humphreys, D.A., Hutchinson, I.H., "Axisymmetric magnetic control design in tokamaks using perturbed equilibrium plasma response modeling," Fus. Tech. 23 (1993) 167.

[9] Penaflor, B.G., Ferron, J.R., Johnson, R.D., Piglowski, D.A., "Current status of DIII-D plasma control system computer upgrades," Proc. 4th IAEA Technical Meeting on Control, Data Acquisition and Remote Participation for Fusion Research, San Diego, California, (2003); to be submitted to Fus. Eng. and Design.

[10] Khayrutdinov, R.R., Lukash, V.E., "Studies of plasma equilibrium and transport in a Tokamak fusion device with the Inverse-Variable Technique," J. Comput. Phys 109 (1993) 193.

[11] Leuer, J.A., et al., "DIII-D plasma control simulation environment," these proceedings.

[12] Callis R.W., Lohr J., Ponce D., Harris T.E., O’Neill R.C., Remsen D.B., Prater R. and Luce T.C., "3 MW, $110 \mathrm{GHz}$ ECH System for the DIII-D tokamak," Proc. 20th Symp. on Fus. Technol., Marseille, France, August 1998, 1 (Association EURATOM-CEA, Saint-PaulLez-Durance, 1999) 315.

[13] Albanese, R., Ambrosino, G., Coccorese, E., Morabito, F.C., Pironti, A., Rubinacci, G., Scala, S., "Plasma current, shape, and position control in ITER," Fus. Tech. 30 (1996) 167.

[14] Walker., M.L., et al., "Some practical control issues on DIII-D and their relevance for ITER," these proceedings. 